\title{
Critical temperatures of the Ising model on Sierpiñski fractal lattices
}

\author{
Michel Perreau ${ }^{1, *}$ \\ ${ }^{1}$ Université de Paris, IUT Paris Pajol, 20 quater rue du département, 75018 Paris, France
}

\begin{abstract}
We report our latest results of the spectra and critical temperatures of the partition function of the Ising model on deterministic Sierpiñski carpets in a wide range of fractal dimensions. Several examples of spectra are given. When the fractal dimension increases (and correlatively the lacunarity decreases), the spectra aggregates more and more tightly along the spectrum of the regular square lattice. The single real root $v_{c}$, comprised between 0 and 1 , of the partition function, which corresponds to the critical temperature $T_{c}$ through the formula $v_{c}=\tanh \left(1 / T_{c}\right)$, reliably fits a power law of exponent $-k$ where $k$ is the segmentation step of the fractal structure. This simple expression allows to extrapolate the critical temperature for $k \rightarrow \infty$. The plot of the logarithm of this extrapolated critical temperature versus the fractal dimension appears to be reliably linear in a wide range of fractal dimensions, except for highly lacunary structures of fractal dimensions close from 1 (the dimension of a quasilinear lattice) where the critical temperature goes to 0 and its logarithm to $-\infty$.
\end{abstract}

\section{Introduction}

Phase transitions on fractals have been investigated since the early applications of fractals in physics, initiated by B. Mandelbrot in three pionnering papers using mainly real space renormalisation group (RSRG) techniques[13]. Subsequently, the Sierpiñski carpet[4] and its extensions have been used as models to test (i) the congruence between critical exponents calculated by the analytical continuation of $\varepsilon$-expansions of the renormalisation approach in non integer dimensions[5] and their values obtained by direct calculations by different methods (ii) the validity of scaling relations involving the critical exponents when replacing the dimension of space by the fractal dimension.

Actually, Sierpiñsky carpets are infinitely ramified, a condition which is mandatory to get a phase transition at non zero temperature and, by varying parameters, their fractal dimensions may approach any desired value as close as necessary. Furthermore, the Ising model is one of the simplest model of phase transitions This lead many authors to combine Sierpiñsky carpets and the Ising model to investigate phase transitions on fractal lattices.

Previous litterature contains many estimations of these critical parameters obtained by different methods: RSRG[1-3, 6, 7]; high temperature expansions [7]; Monte-Carlo simulations either with the Metropolis or with the Wolf or the Swendsen-Wang algorithms combined with a finite size scaling analysis (MCFS) [8-17]; Monte-Carlo simulations combined with renormalisation group (MCRG) [18]; Monte-Carlo simulations combined with short time dynamic scaling (MCSD) [19-22]. According to references, spins are located either on the center of the sites [11-17, 20-22] or on the vertices of the lattice

*e-mail: michel.perreau@u-paris.fr
$[1-3,6-11,19,23]$. The results presented in this work are obtained with spins located in the center of the sites, and so we will compare critical paramers only within this topology.

The most accurate values of critical exponents $\beta$ and $\gamma$ have been obtained by the various methods involving Monte Carlo simulations and show that values of critical exponents are different than those obtained by continuation of $\varepsilon$-expansions. So replacing translation invariance by scale invariance changes significantly the critical behavior. But scaling relations - at least those which do not involve $\alpha$, the estimation of which is unaccurate in fractals[13] - as the Rushbrooke and Josephson's scaling law: $d v=\gamma+2 \beta$ are satisfied within the accuracy of these numerical simulations $[12,15]$

However, Pruessner and Loison[16] questioned the relevance of finite size scaling performed on the successive segmentation steps of a single pattern, arguing that the size of the structures is not large enough to exceed the correlation length in the critical region. Alternatively, they based their calculations by reintroducing translation invariance with the juxtaposition of several identical patterns in the two dimensions of space to increase the size of the elementary cell before applying the usual periodic boundary conditions. For a scale invariance $n=3$ with a single centered removed site, the values of the critical temperatures obtained after this modification are close to those obtained previously, but significantly different according to their respective accuracy, 1.50 instead of 1.48. This significant discrepancy reveals the difficulty of calculating accurate values of the critical temperature which is crucial for using histogram and finite size scaling method after Monte Carlo simulations.

The aim of this paper is to push forward the method previously introduced[24] to calculate the critical temper- 
atures the Ising model on Sierpiñski carpets by investigating a larger range of structures and higher segmentation steps to increase the accuracy of results.

\section{The Sierpiñsky carpets}

Let us remind the fractal structures corresponding to Sierpiñski carpets. Starting from an initial unit size square, divided into $n^{2}$ subsquares of equal size we remove $p^{2}$ subsqures in the center and iterate the processus $k$ times. This structure in noted $S C(n, p, k)$. The fractal structure $S C(n, p)$ is the limit of this process when $k \rightarrow \infty$. Fig. 1 shows the third segmentation step of $S C(3,1)$, i.e. $S C(3,1,3)$. Ising spins are located on center of the remaining sites.

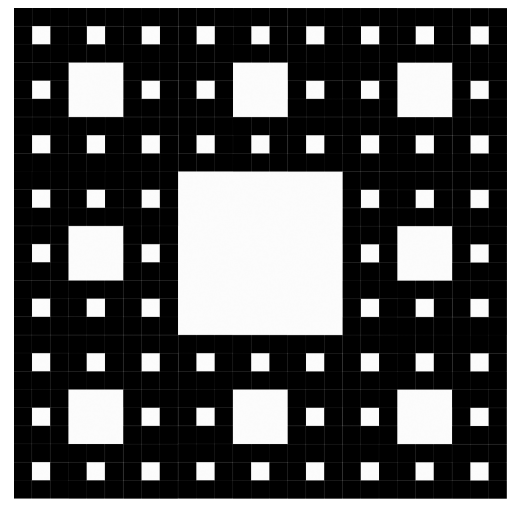

Figure 1. The third segmentation step of $S C(3,1)$

\section{Ising model on Sierpiñski carpets}

\subsection{Partition functions}

In a previous paper[24] we introduced a method to calculate the partition function of the Ising model in planar lacunary and especially fractal lattices, at any segmentation step. We could calculate the full spectrum (i.e. the set of the roots) of this partition function on Sierpiñski carpets and particularly the critical temperature $T_{c}$ which corresponds to the single real value $v_{c}$ of the spectrum comprised between 0 and 1 through the formula

$$
v_{c}=\tanh \left(1 / T_{c}\right)
$$

The method is an extension of the path counting method used to calculate the partition function of the two dimensional Ising model on square lattice[25]. The aim of this paper is not to expose again the method, but to give more accurate results based on further calculations in the three fields which can be investigated in this way on planar fractal lattices: (i) the partition functions and its spectrum (ii) the critical temperatures on finite values of the segmentation step $k$ and an extended set of fractal dimensions (or equivalently of values of parameters $n$ and $p$ defined above), (iii) the extrapolation from the sequences of critical temperatures at increasing values of the segmentation step $k$ of the critical temperature at the actual fractal limit $k \rightarrow \infty$.

Generally, the partition function is expressed by a polynomial $\mathcal{P}(N, v)$ which counts the closed pathes on the lattice:

$$
Z=2^{N} \cosh \left(\frac{1}{T}\right)^{N_{z}} \mathcal{P}(N, v)
$$

where $N$ is the total number of patterns, $N_{z}$ is the number of neighbours, $v=\tanh (1 / T)$.

For two dimensional lattices, $\mathcal{P}$ is obtained as the characteristic polynomial of an appropriate matrix[24]. For example, for $S C(3,1,1)$, the first segmentation step of the most simple Sierpiñski carpet, this polynomial is

$$
\begin{aligned}
\mathcal{P}(N, v)= & \prod_{p, q=1}^{N}\left\{Q_{1}(v)+Q_{2}(v)\left[\cos \left(\frac{2 \pi p}{N}\right)+\cos \left(\frac{2 \pi q}{N}\right)\right]\right. \\
& \left.+Q_{3}(v) \cos \left(\frac{2 \pi p}{N}\right) \cos \left(\frac{2 \pi q}{N}\right)+Q_{4}(v)\left[\cos \left(\frac{2 \pi p}{N}\right)^{2}+\cos \left(\frac{2 \pi q}{N}\right)^{2}\right]\right\}^{\frac{1}{2}}
\end{aligned}
$$

with

$$
\begin{aligned}
& Q_{1}(v)=\left(1+v^{2}\right)^{2}\left(1-2 v^{2}+13 v^{4}-8 v^{6}+126 v^{8}+108 v^{10}\right. \\
& \left.+474 v^{12}+248 v^{14}+57 v^{16}+6 v^{18}+v^{20}\right) \\
& Q_{2}(v)=-4 v^{3}\left(1-v^{2}\right)^{2}\left(1+3 v^{2}\right)\left(1+v^{2}+2 v^{4}\right) \\
& \left(1+2 v^{2}+8 v^{4}+4 v^{6}+v^{8}\right) \\
& Q_{3}(v)=-4 v^{6}\left(1-v^{2}\right)^{4}\left(1+v^{2}\right)\left(7+11 v^{2}+13 v^{4}+v^{6}\right) \\
& Q_{4}(v)=+4 v^{6}\left(1-v^{2}\right)^{5}\left(1+v^{2}\right)\left(1+3 v^{2}\right)
\end{aligned}
$$

The critical temperature is given by the real positive root of $P(v)=\mathcal{P}(1, v)$ :

$$
P(v)=1-4 v^{3}+5 v^{4}-16 v^{5}-10 v^{6}-20 v^{7}+v^{8}-24 v^{9}+2 v^{10}+v^{12}
$$

which is $v_{c} \approx 0.4960$ giving $T_{c}=1 / \operatorname{Atanh}\left(v_{c}\right) \approx 1.8384$.

The polynomial $P(v)$ can be factorized through rotation invariance in

$$
P(v)=Q^{+}(v) \cdot Q^{-}(v)
$$

with

$$
Q^{ \pm}(v)=1+v^{2}+5 v^{4}+v^{6} \pm \sqrt{2} v\left(1+v+v^{2}+3 v^{3}+2 v^{4}\right)
$$

Since all coefficients of $Q^{+}$are positive, it cannot have positive real roots, so $v_{c}$ is a root of $Q^{-}$.

\subsection{Spectra}

Some exemples of spectra are shown on Figs. 2-6 for different values of $n$ and $p: p=n-2$ (Fig. 2), $p=n-4$ (Fig. 3), $p=n-6$ (Fig. 5), $p=n-8$ (Fig. 6). The two red circles are the location in which the spectrum of the partition function of the Ising model on the square lattice is dense[26].

We observe that more the structure is compact, with a lower lacunarity (increasing values of $n-p$ or increasing concentration $n^{2} / p^{2}$ ), then the spectrum aggregates more tightly along these two circles.

Fig. 4 shows a magnification of the region of the spectrum of $S C(3,1, k)$ located near the real axis (delimited by the blue frame on the top of Fig. 2), for $k=5$ and $k=6$. It appears that despite being spectra of scale invariant fractal structures and being themselves likely fractals, these spectra are not scale invariant. 

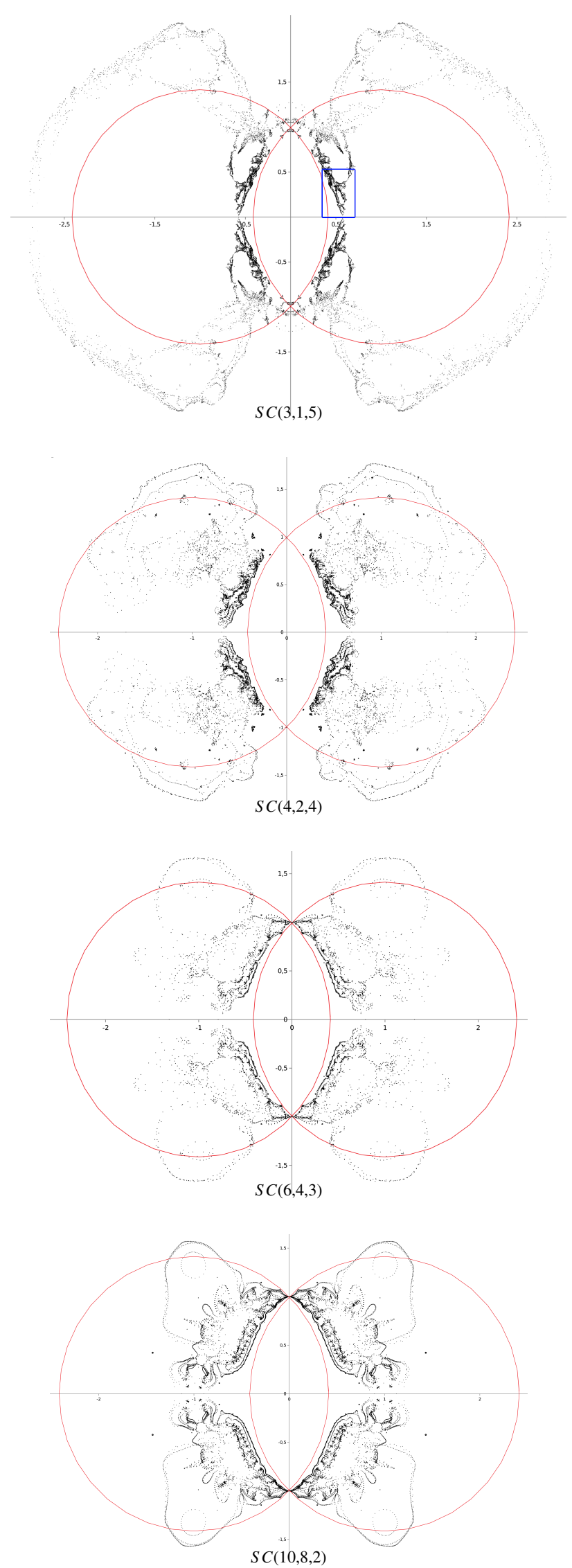

Figure 2. Spectra of the Ising model on $S C(n, p, k)$ for $p=n-2$.
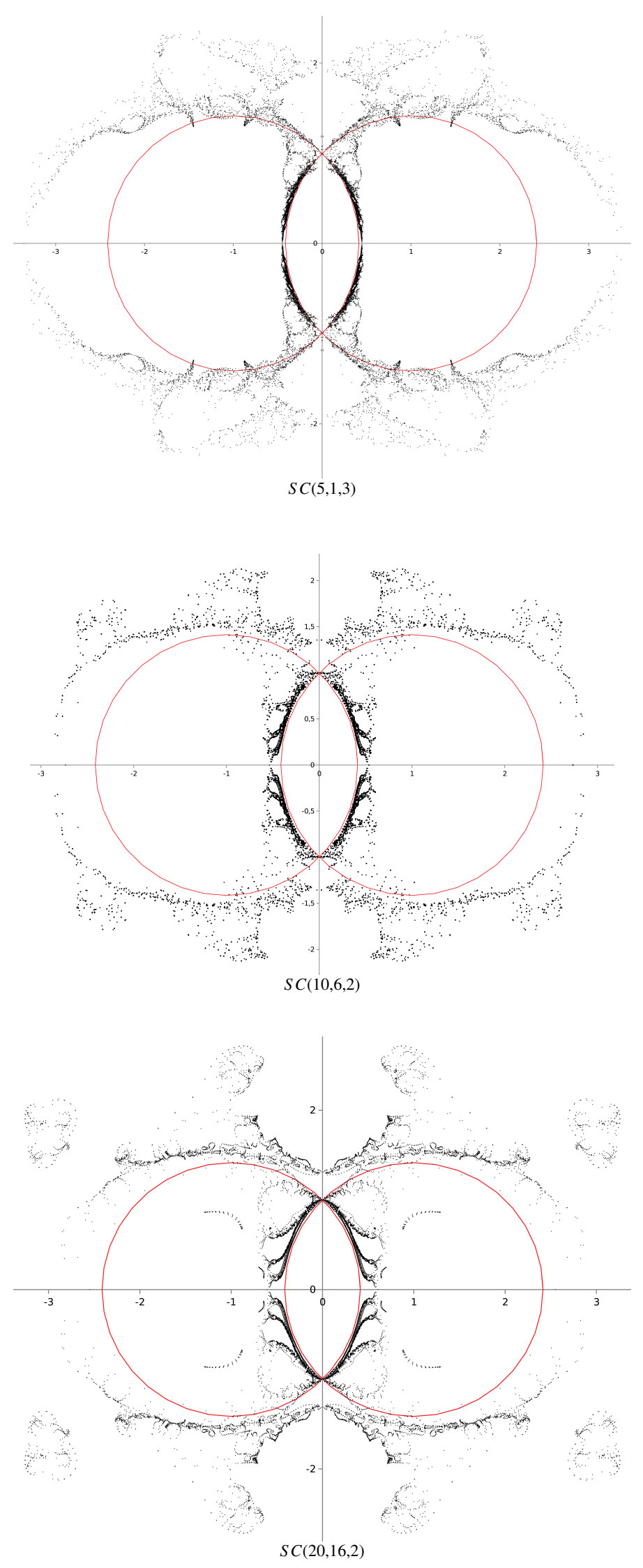

Figure 3. Spectra of the Ising model on $S C(n, p, k)$ for $p=n-4$. 


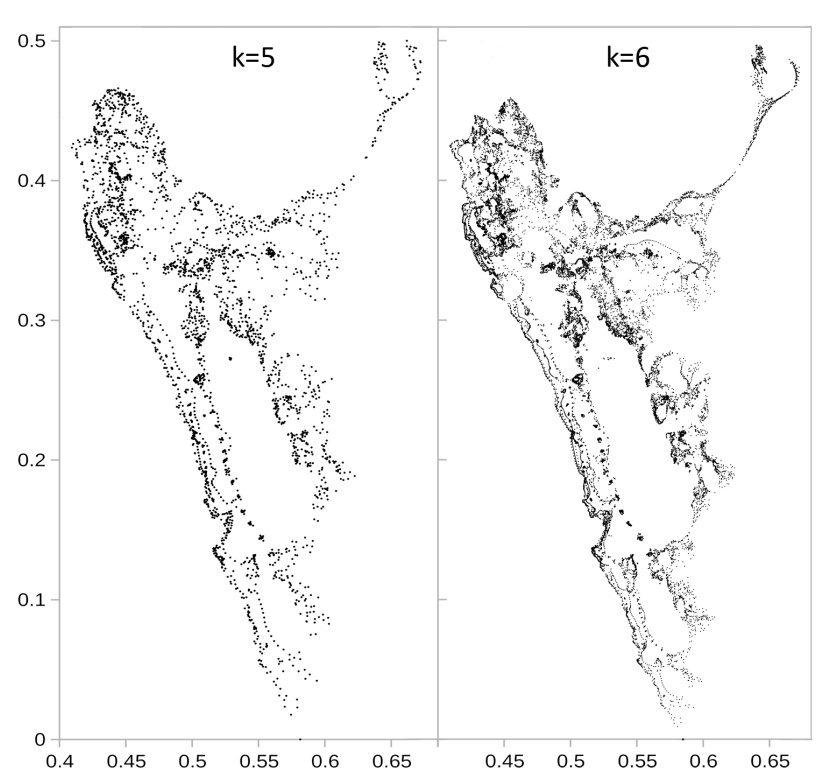

Figure 4. Spectrum of $S C(3,1, k)$ in the vicinity of the real axis (magnification of the blue rectangle of Fig. 2).

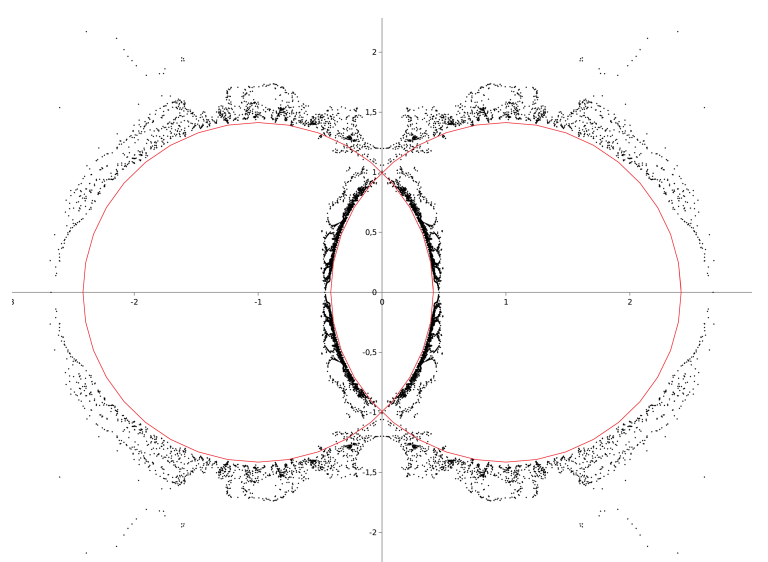

$S C(10,4,2)$

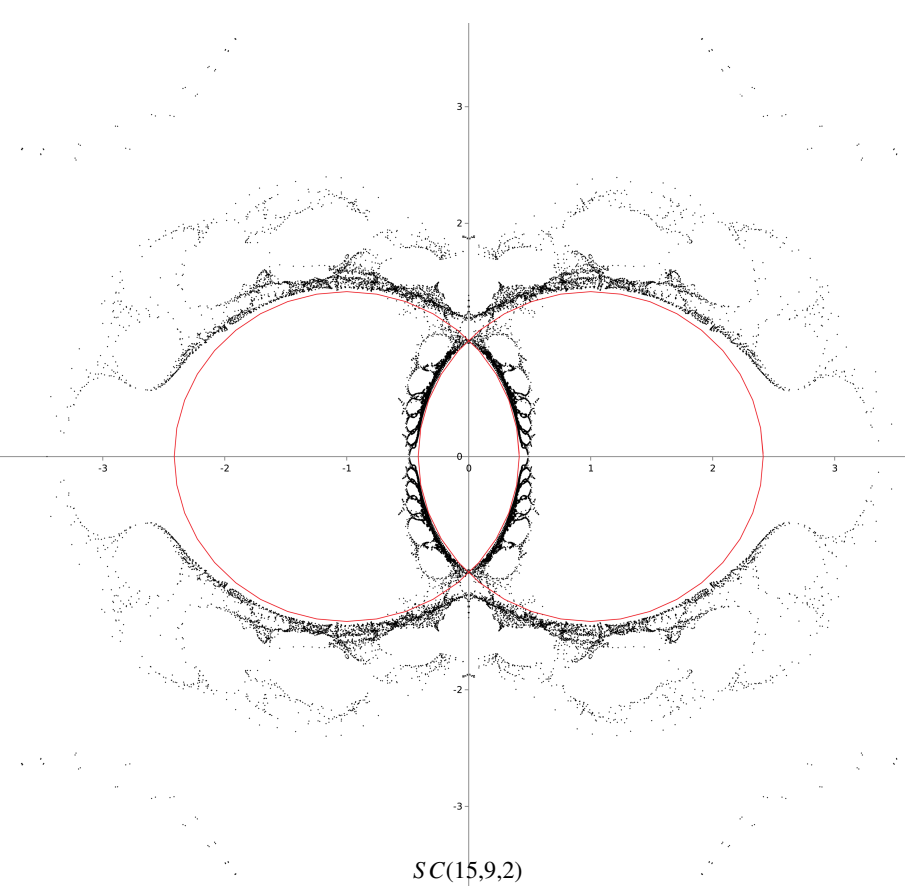

Figure 5. Spectra of the Ising model on $S C(n, p, k)$ for $p=n-6$.
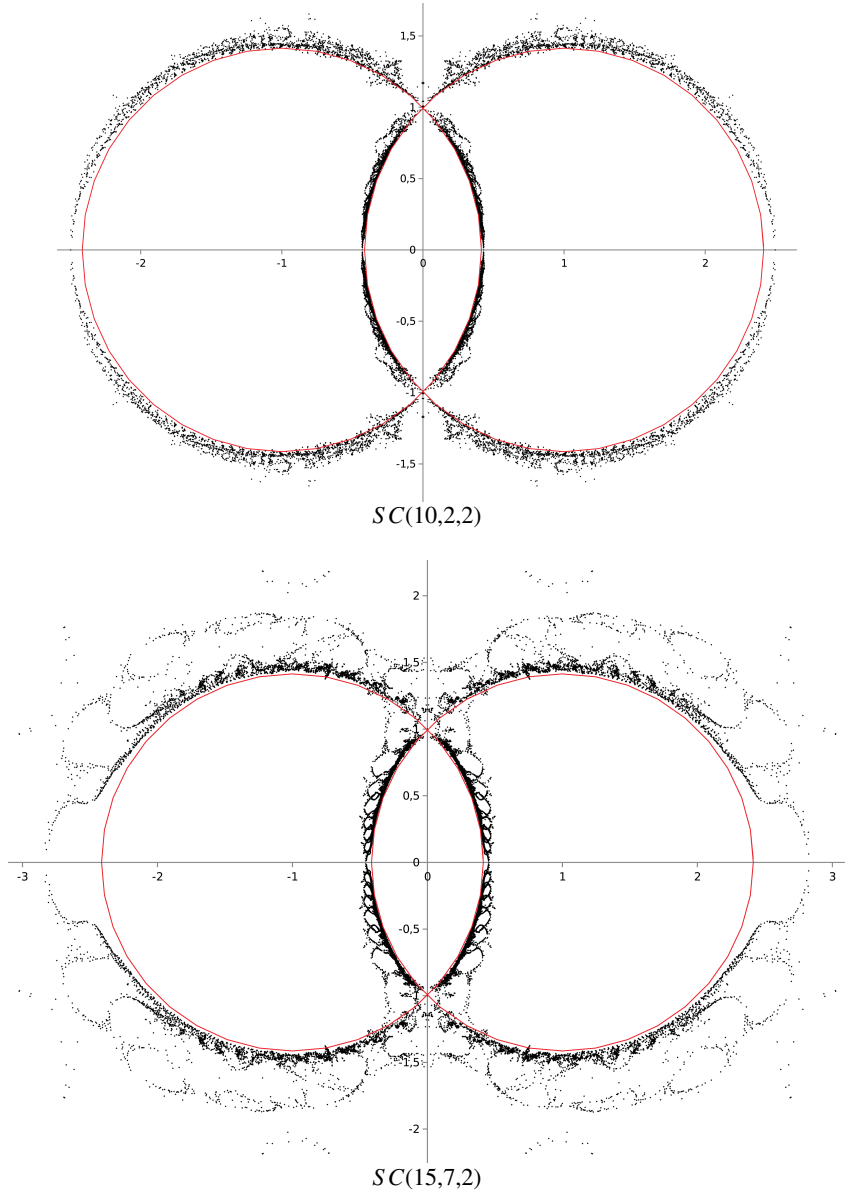

Figure 6. Spectra of the Ising model on $S C(n, p, k)$ for $p=n-8$.

\subsection{Critical temperatures}

The values of $v_{c}$ have been calculated for a wide range of structures, up to $n=100$ and $p=n-2$ to $p=n-8$. We will not give the whole details of updated data. But denoting $v_{c s q}=\sqrt{2}-1$, the value of $v_{c}$ for the square lattice, the plots of $v_{c}-v_{c s q}$, recorded in Fig. 7 for $p=n-2, p=n-4$, $p=n-6$ and $p=n-8$ fit quite reliably a power law of exponent $-k$ :

$$
v_{c}-v_{c s q}=v_{c}-\sqrt{2}+1=a(n, p)\left[1-b(n, p)^{-k}\right]
$$

Dotted lines in Fig. 7 correspond to least square linear fits. The values of $a(n, p)$ and $b(n, p)$ are chosen to maximize the determination coefficients $R^{2}$ of the least square linear fits for each value of $n$ and $p$. All $R^{2}$ differ from 1 by less than $10^{-4}$.

All values of $b(n, p)$ are greater than 1 , then $v_{c}-v_{c s q}$ converges towards $a(n, p)$ when $k \rightarrow \infty$, leading to the extrapolation of $v_{c}$, and then of $T_{c}$, when $k \rightarrow \infty$. The values of $a(n, p), b(n, p), T_{c}(k=\infty)$ and the maximum value of the segmentation step $k_{\max }$ on which extrapolations have been performed, are given in Table 1 for all investigated Sierpiñski carpets up to $n=100$. The structures corresponding to the three highest values of $n$ for $p=n-2$ : $n=25, n=50$ and $n=100$ are so lacunary that the accuracy of the extrapolations is not enough to distinguish the critical temperatures from $T_{c}=0$. The plots of $b(n, p)$ are given on Fig. 8 (on the right). 
Table 1. Values of $a, b$ of eq. (1) and of $T_{c}(k=\infty)$ (enlighted in boldfaces), $k_{\max }$ is the highest segmentation step used for calculations.

\begin{tabular}{|c|c|c|c|c|c|c|c|c|c|c|c|c|c|c|c|c|}
\hline & \multicolumn{4}{|c|}{$p=n-2$} & \multicolumn{4}{|c|}{$p=n-4$} & \multicolumn{4}{|c|}{$p=n-6$} & \multicolumn{4}{|c|}{$p=n-8$} \\
\hline$n$ & $a$ & $b$ & $T_{c}(\infty)$ & $k_{\max }$ & $a$ & $b$ & $T_{c}(\infty)$ & $k_{\max }$ & $a$ & $b$ & $T_{c}(\infty)$ & $k_{\max }$ & $a$ & $b$ & $T_{c}(\infty)$ & $k_{\max }$ \\
\hline 3 & 0,1745 & 1.895 & 1.4800 & 8 & & & & & & & & & & & & \\
\hline 4 & 0.3053 & 1.780 & 1.1030 & 5 & & & & & & & & & & & & \\
\hline 5 & 0.4056 & 1.721 & 0.8648 & 5 & 0.0355 & 3.573 & 2.0649 & 4 & & & & & & & & \\
\hline 6 & 0.4622 & 1.763 & 0.7351 & 4 & 0.0628 & 3.761 & 1.9266 & 4 & & & & & & & & \\
\hline 7 & 0.4998 & 1.810 & 0.6447 & 4 & 0.0846 & 3.811 & 1.8259 & 3 & 0.0154 & 5.130 & 2.1766 & 3 & & & & \\
\hline 8 & 0.5237 & 1.871 & 0.5813 & 4 & 0.1025 & 3.843 & 1.7489 & 3 & 0.0286 & 5.562 & 2.1024 & 3 & & & & \\
\hline 9 & 0.5393 & 1.936 & 0.5396 & 4 & 0.1177 & 3.854 & 1.6868 & 3 & 0.0394 & 5.805 & 2.0441 & 3 & 0.0086 & 6.686 & 2.2165 & 3 \\
\hline 10 & 0.5481 & 2.001 & 0.5061 & 4 & 0.1309 & 3.858 & 1.6355 & 3 & 0.0486 & 5.947 & 1.9964 & 3 & 0.0165 & 7.339 & 2.1704 & 3 \\
\hline 11 & 0.5606 & 2.037 & 0.4583 & 3 & 0.1425 & 3.861 & 1.5921 & 3 & 0.0565 & 6.141 & 1.9571 & 3 & 0.0232 & 7.659 & 2.1320 & 3 \\
\hline 12 & 0.5666 & 2.095 & 0.4313 & 3 & 0.1528 & 3.864 & 1.5548 & 3 & 0.0636 & 6.167 & 1.9224 & 3 & 0.0290 & 7.915 & 2.0997 & 3 \\
\hline 13 & 0.5708 & 2.153 & 0.4091 & 3 & 0.1621 & 3.868 & 1.5224 & 3 & 0.0701 & 6.133 & 1.8917 & 3 & 0.0342 & 8.089 & 2.0717 & 3 \\
\hline 14 & 0.5741 & 2.210 & 0.3897 & 3 & 0.1704 & 3,874 & 1.4938 & 3 & 0.0759 & 6.164 & 1.8651 & 3 & 0.0388 & 8.213 & 2.0472 & 3 \\
\hline 15 & 0.5765 & 2.266 & 0.3725 & 3 & 0.1780 & 3.880 & 1.4684 & 3 & 0.0813 & 6.190 & 1.8413 & 3 & 0.0430 & 8.305 & 2.0253 & 2 \\
\hline 20 & 0.5832 & 2.524 & 0.3001 & 3 & 0.2077 & 3.920 & 1.3732 & 3 & 0.1019 & 6.265 & 1.7514 & 2 & 0.0594 & 8.558 & 1.9427 & 2 \\
\hline 25 & $0.59 ?$ & 2.754 & $\sim 0$ & 3 & 0.2288 & 3.978 & 1.3101 & 2 & 0.1167 & 6.355 & 1.6909 & 2 & 0.0712 & 8.702 & 1.8867 & 2 \\
\hline 50 & $0,60 ?$ & 3.429 & $\sim 0$ & 2 & 0.2861 & 4.182 & 1.1521 & 2 & 0.1575 & 6.654 & 1.5385 & 2 & 0.1033 & 9.157 & 1.7454 & 2 \\
\hline 100 & $0.59 ?$ & 4.686 & $\sim 0$ & 2 & 0.3343 & 4.425 & 1.0314 & 2 & 0.1928 & 6.923 & 1.4200 & 2 & 0.1170 & 9.555 & 1.6362 & 2 \\
\hline
\end{tabular}
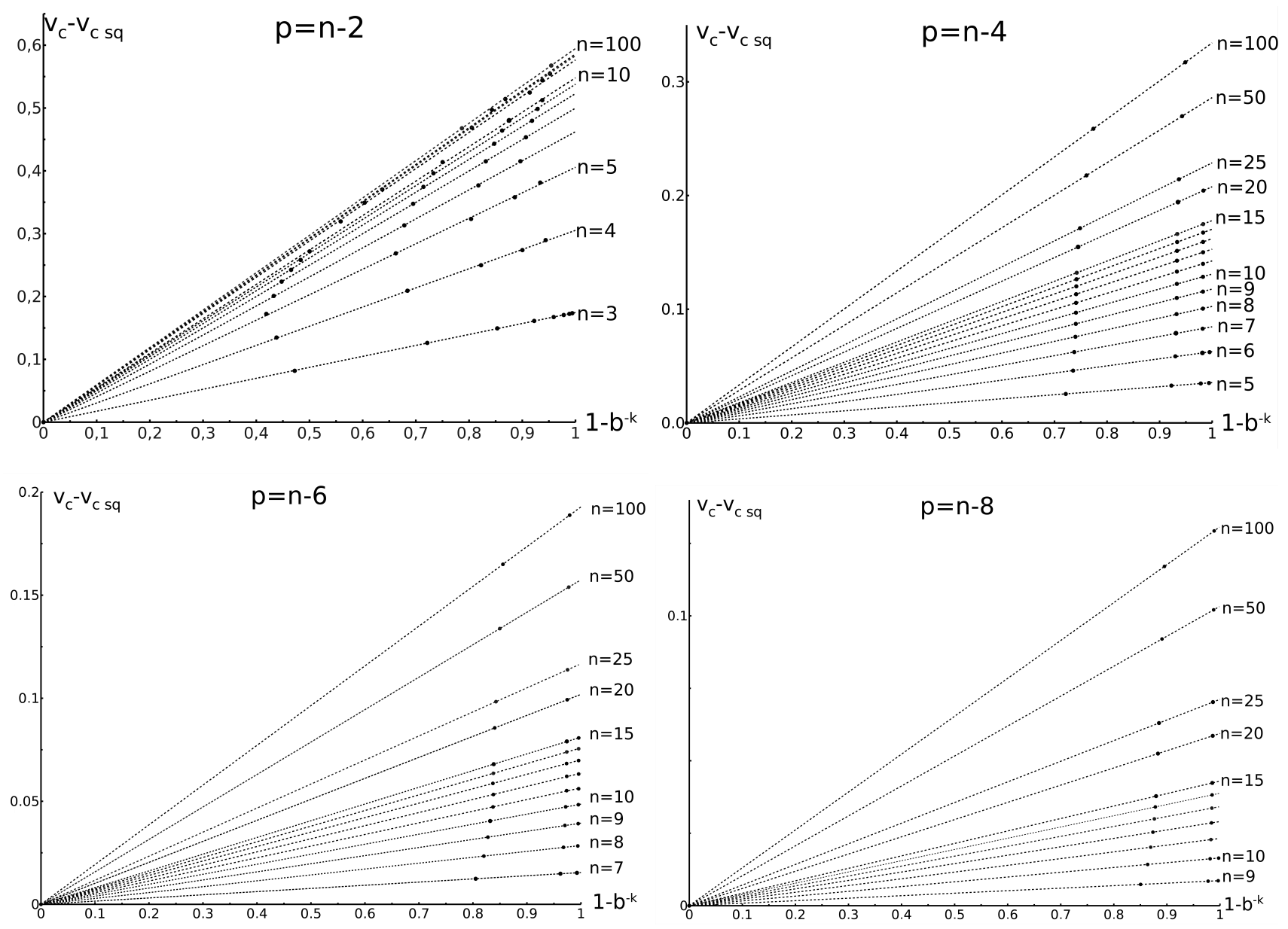

Figure 7. Plots of $v_{c}-v_{c s q}$ versus $\left(1-b^{-k}\right)$ for Sierpiñski carpets corresponding to a wide range of values of $n$ and $p$. All plots are linear after an appropriate choice of $a$ and $b$.

Once extrapolated, it is possible to investigate the variations of $T_{c}(\infty)$ versus the fractal dimension $d_{h}$. The plots of $\log \left[T_{c}(\infty)\right]$ versus $d_{h}$ for $p=n-2$ to $n-8$ are shown on Fig. 8 (on the left). The curves are reliably linear in a wide domain which excludes the vicinity of the fractal dimension 1, corrresponding to quasilinear lattices, where is is known that $T_{c}(\infty)=0[1]$ and then $\log \left[T_{c}(\infty)\right]=-\infty$, and at the other extremity, in a close vicinity of $d=2$, 


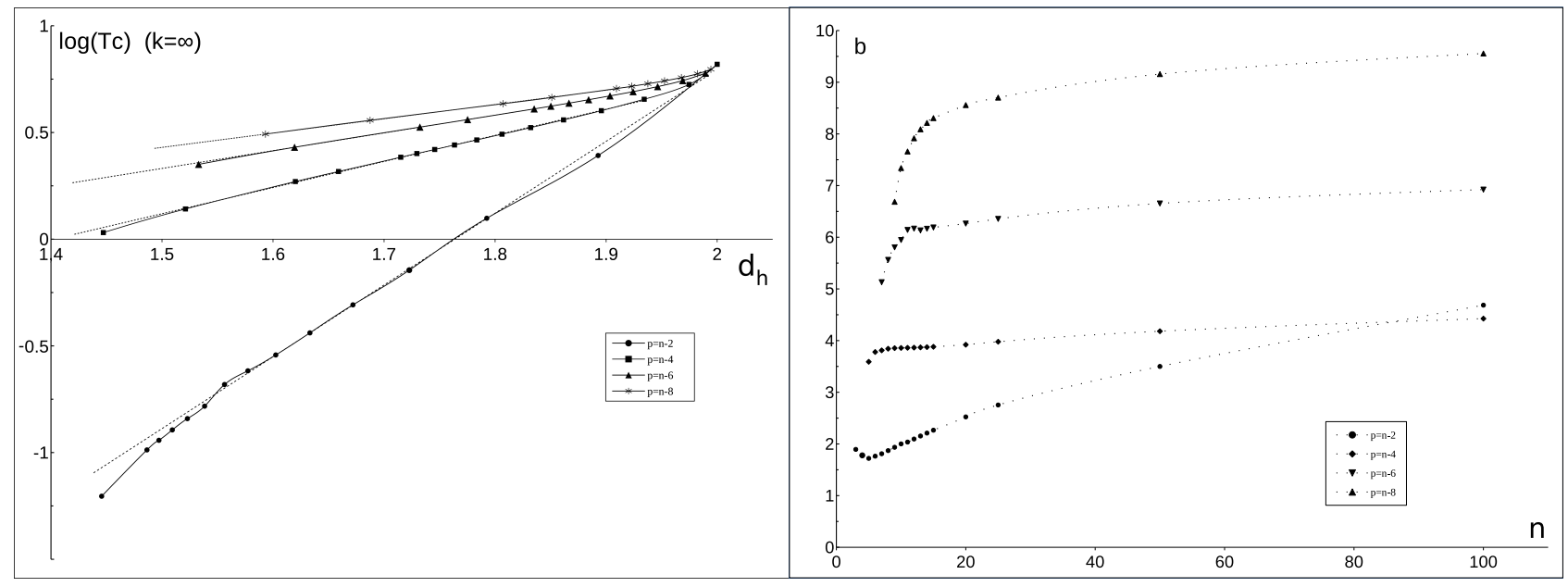

Figure 8. Plots of $\log \left[T_{c}(\infty)\right]$ versus $d_{h}$, dotted lines are linear fits (left) and $b(n, p)$ versus $n$ for $p=n-2, p=n-4, p=n-6$, $p=n-8$ (right)

corresponding to the square lattice. The determination coefficients $R^{2}$ of the least square linear fits differ from 1 by less than $10^{-4}$ for $p=n-4, p=n-6$ and $p=n-8$, less than $10^{-3}$ for $p=n-2$.

The linear parts of these plots lead to the following power laws of the critical temperature versus the fractal dimension:

$$
\begin{array}{ll}
\text { for } \quad p=n-2, & T_{c}(\infty) \approx 2.610^{-3}(28.9)^{d_{h}} \\
\text { for } \quad p=n-4, & T_{c}(\infty) \approx 0.180(3.397)^{d_{h}} \\
\text { for } \quad p=n-6, & T_{c}(\infty) \approx 0.400(2.298)^{d_{h}} \\
\text { for } \quad p=n-8, & T_{c}(\infty) \approx 0.563(1.990)^{d_{h}}
\end{array}
$$

\section{Erratum of a previous reference}

By the way we would proceed here to the corrections of some typos and undesired mistakes which occurred in ref.[24] :

\section{1 - Table 1, last line, MCST should be MCSD}

2 - Page $6, \pi / 4$ should be replaced by $\pi / 2$ three lines before and two lines after the title of the section "Rotational invariance" (two occurrences). The smallest rotation which leaves invariant the square is $\pi / 2$.

3 - Page 7, eq. 11 should be $v_{c}-v_{c s q}=a\left(1-b^{-k}\right)$ (eq. 1 of the present paper) instead of $v_{c}-v_{c s q}=a\left(1+b^{-k}\right)$. The same should be changed in four occurrences in fig. 8.

4 - Page 9, in fig. 10, the graphic attributed to $S C(20,16,2)$ is not the spectrum but the inverse of the spectrum (cf. Fig. 3 of the present paper).

5 - Page 8, in fig. 9, in caption, left and right are inverted. 6 - Page 10, in the formula of the internal energy $U$, in the first line, 128 should be replaced by $2(2 n-1)$ and in the second line, the argument of the elliptic function $K$ should contain $\left(1-v^{2 n}\right)$ instead of $\left(v^{2 n}-1\right)$.

\section{References}

[1] Y. Gefen, B. B. Mandelbrot, and A. Aharony, Phys. Rev. Lett., 45, 855 (1980).
[2] Y. Gefen, Y. Meir, B. B. Mandelbrot, and A. Aharony, Phys. Rev. Lett., 50, 145 (1983).

[3] Y. Gefen, A. Aharony, and B. Mandelbrot, J. Phys. A, 17, 1277 (1984).

[4] W. Sierpiński, Matematitcheskii Sbornik, 30: 267 (1916).

[5] J. C. Le Guillou and J. Zinn-Justin, J. Phys., 49, 19 (1987).

[6] B. Bonnier, Y. Leroyer, and C. Meyers, Phys. Rev. B, 37, 5205 (1988).

[7] B. Bonnier, Y. Leroyer, and C. Meyers, Phys. Rev. B, 40, 8961 (1989).

[8] G. Bhanot, H. Neuberger, and J. A. Shapiro, Phys. Rev. Lett. 53, 2277 (1984).

[9] G. Bhanot, D Duke, and R. Salvador, Phys. Lett, 165B, 355 (1985).

[10] J. C. Angles d'Auriac and R. Rammal, J. Phys. A, 19, L655 (1986).

[11] B. Bonnier, Y. Leroyer, and C. Meyers, J. Phys. (Paris), 48, 553 (1987).

[12] J. Carmona, U. M. B. Marconi, J. J. Ruiz-Lorenzo, and A. Tarancón, Phys. Rev. B, 58, 14387 (1998).

[13] P. Monceau, M. Perreau, and F. Hébert, Phys. Rev. B, 58, 6386 (1998).

[14] P. Y. Hsiao, P. Monceau, and M. Perreau, 2000, Phys. Rev. B, 62: 13856.

[15] P. Monceau and M. Perreau, Phys. Rev. B, 63: 184420-184429 (2001).

[16] G. Pruessner, D. Loison, and K. D. Schotte, Phys. Rev. B, 64, 134414 (2001).

[17] P. Monceau and P. Y. Hsiao, 2004, Physica A, 331: 1 (2004).

[18] P. Y. Hsiao and P. Monceau, Phys. Rev. B, 67: 064411 (2003).

[19] G. P. Zheng and M. Li, Physica A 283, 80 (2000).

[20] M. A. Bab, G. Fabricius, and E. V. Albano, Phys. rev. E 71, 036139 (2005). 
[21] M. A. Bab, G. Fabricius, and E. V.Albano, Phys. rev. E 74, 041123 (2006).

[22] M. A. Bab, G. Fabricius, and E. V. Albano, Physica A 388, 370 (2009).

[23] A. Venezzani, J. Phys. A, 36, 1593 (2003)

[24] M. Perreau, Phys. Rev. B, 96: 174407 (2017)
[25] M. Kac and J. C. Ward, Phys. Rev., 88 (6), 1332 (1952).

[26] B. M. McCoy and T. T. Wu, The Two-Dimensional Ising Model. (Harvard University Press, Cambridge Massachusetts, 1973), $441 \mathrm{pp}$. 